\title{
Influencia del proceso de revenido en el comportamiento mecánico de un acero de fase dual de uso industrial automotriz
}

\author{
Influence of tempering process on the mechanical behavior of dual phase steels \\ for automotive applications
}

\author{
Rodolfo Rodríguez-Baracaldo $^{1} \quad$ José Manuel Arroyo-Osorio $^{1} \quad$ Yeison Parra-Rodríguez $^{1}$
}

Recibido 12 de noviembre de 2014, aceptado 2 de abril de 2015

Received: November 12, $2014 \quad$ Accepted: April 2, 2015

\begin{abstract}
RESUMEN
En el presente trabajo se analizó la influencia del tratamiento del revenido en la microestructura y en las propiedades mecánicas del acero de fase dual ferrita-martensita de alta resistencia DP580. Las transformaciones microestructurales analizadas fueron el resultado de aplicar el proceso de revenido en un rango de temperatura de 150 a $500{ }^{\circ} \mathrm{C}$. El análisis metalográfico y fractográfico del acero inicial y el tratado térmicamente se realizó con microscopia óptica y electrónica. Los cambios en las propiedades mecánicas se evaluaron mediante ensayos de tracción uniaxial y microdureza. Se observó que el rango de temperaturas de revenido utilizado afecta de manera gradual la microestructura permitiendo obtener acero con diferentes combinaciones de resistencia y ductilidad. Los efectos del tratamiento térmico de revenido sobre el acero de fase dual analizado son consecuencia de un efecto sinérgico entre las transformaciones en la fase martensita y en la fase ferrita.
\end{abstract}

Palabras clave: Aceros de fase dual, revenido, propiedades mecánicas.

\begin{abstract}
In this paper was analyzed the influence of annealing treatment on the microstructure and mechanical properties of ferrite-martensite of dual phase of high strength steel DP580. The analyzed microstructural transformations were the result of applying the annealing process in a temperature range of 150 to 500 ${ }^{\circ}$ C. Metallographic and fractographic analysis were performed on the initial steel and heat-treated steel by using optical and electron microscopy. Modifications in mechanical properties were evaluated by uniaxial tensile tests and by microhardness tests. It was observed that the used range of annealing temperatures affects gradually the microstructure, allowing obtaining different combinations of steel strength and ductility. The effects of annealing heat treatment on the dual phase steel analyzed are the result of a synergistic effect between the transformations in the martensite phase and ferrite phase.
\end{abstract}

Keywords: Dual phase steel, tempering, mechanical properties.

\section{INTRODUCCIÓN}

En los últimos años ha aumentado el interés en el desarrollo y aplicación industrial de los denominados aceros avanzados de alta resistencia (AHSS-advanced high strength steels) [1-3].
Entre este grupo de materiales se destacan los aceros de fase dual (DP-dual phase steels), los cuales poseen una matriz ferrítica integrada con una segunda fase martensítica. Esta combinación de fases permite obtener simultáneamente elevada resistencia mecánica y apreciable ductilidad. Esta

1 Departamento de Ingeniería Mecánica y Mecatrónica. Universidad Nacional de Colombia. Carrera 45 N ${ }^{\circ}$ 26-85. Bogotá, Colombia.E-mail: rodriguezba@unal.edu.co; jmarroyoo@unal.edu.co; yparrar@unal.edu.co 
combinación de propiedades es escasa entre los aceros convencionales y puede obtenerse con aceros aleados pero a un costo elevado. Los aceros DP son menos costosos que los aceros aleados presentando propiedades similares [4-5]. Con los aceros DP es posible diseñar componentes estructurales con espesores más delgados, lo que se traduce en economía de material y menor peso. El uso de aceros DP en la fabricación de vehículos automotores permite, además del ahorro en consumo de materiales, un importante ahorro de combustible en la operación del vehículo por la disminución de su peso global. Otro beneficio es el incremento de la seguridad de los pasajeros debido también a la mejoría en el desempeño ante el impacto de los aceros DP [3, 6-7].

Los aceros DP se obtienen mediante tratamientos térmicos a temperaturas intercríticas. En el caso de los aceros hipoeutectoides se realiza el proceso calentamiento hasta una temperatura en el rango entre Ac1 y Ac3, seguido de un proceso de enfriamiento controlado para transformar la austenita en martensita $[2,5]$. El control de las variables del tratamiento permite el control indirecto del tamaño, la distribución y la cantidad de martensita en la matriz ferrítica [4, 8]. La fase blanda ferrítica es generalmente continua y volumétricamente importante. La deformación del acero se facilita gracias a que esta fase se concentra justo alrededor de las islas de martensita, presentando una excelente ductilidad y alta tasa de endurecimiento por deformación. Varios estudios [8-9] han demostrado que las características de la martensita son el factor determinante en la resistencia mecánica del acero. Adicionalmente otros trabajos se han concentrado en los factores que influyen en la ductilidad de los aceros DP, entre otros, la presencia de austenita retenida o la composición química de la martensita y de la ferrita [6, 10-12].

La respuesta de los aceros DP a los tratamientos térmicos ha sido estudiada por varios autores. Eldis [13] investigó el efecto del revenido en aceros DP con diferencias en la composición química, así como la cantidad de austenita retenida para una relación de ferrita - martensita determinada. Rashid y Rao [14] y también Luo y colaboradores [15] estudiaron los precipitados formados en el acero en función de la temperatura de revenido empleada. Estos autores encontraron que los carburos $\varepsilon$ se empiezan a precipitar cuando la temperatura de revenido se incrementa y también estudiaron la evolución de los carburos con los gradientes de temperatura. Matsuda y colaboradores [16] estudiaron los efectos del comportamiento de autorevenido de la martensita en las propiedades mecánicas de láminas de acero de ultra alta resistencia.

Un aspecto importante del uso de los aceros DP para aplicaciones estructurales en fabricación de vehículos es su conformabilidad. Una alternativa para mejorar la conformabilidad es aplicar procesos de revenido, pero debe evaluarse también el efecto de estos procesos en la resistencia a fluencia y en la resistencia máxima del acero. Con el objetivo de evaluar la posibilidad de ajustar las propiedades de los aceros DP por medio del tratamiento térmico de revenido, en el presente trabajo se analizó la influencia de este tratamiento en el rango de temperatura de 150 a $500{ }^{\circ} \mathrm{C}$ sobre la microestructura, la resistencia mecánica, la dureza de las fases y la ductilidad del acero de fase dual ferrita-martensita de alta resistencia DP580.

\section{PROCEDIMIENTO EXPERIMENTAL}

Para el ensayo de revenido, se emplearon probetas de lámina de acero DP580 de 70,0×12,7×3,05 $\mathrm{mm}$, con composición química en $\%$ peso de 0,069 C, 0,688 Si, 1,216 Mn, 0,0009 P, 0,007 S y balance Fe. Este acero tiene una estructura de fase dual ferrita-martensita obtenida mediante tratamiento de recocido intercrítico en la región austenita-ferrita con enfriamiento rápido posterior hasta la temperatura ambiente para transformar la austenita en martensita. Las probetas se sometieron a un proceso de revenido en atmosfera controlada a temperaturas de $150,200,300,400$ y $500{ }^{\circ} \mathrm{C}$ durante 1 hora seguido de enfriamiento al aire. Gündüz [17], Addalla y colaboradores [18] y Fang y colaboradores [19], mostraron que para este tipo de aceros, temperaturas de revenido superiores a $500{ }^{\circ} \mathrm{C}$ afectan negativamente las propiedades mecánicas; mientras temperaturas de revenido por debajo de $150{ }^{\circ} \mathrm{C}$ no tienen efecto evidente sobre la microestructura y las propiedades. Estos autores determinaron también que para el rango de temperaturas seleccionado es suficiente un tiempo de revenido de una hora.

La microestructura del acero antes y después del proceso de revenido fue observada con 
un microscopio electrónico de barrido (MEB) FEI_Quanta 200 y también con un microscopio óptico Leco 500. Para realizar la observación por microscopía, las probetas fueron previamente atacadas con solución de nital al 4\%. El cálculo de la fracción volumétrica de fases se realizó utilizando el software de análisis de imágenes IA32 sobre las fotografías obtenidas tanto con microscopía óptica como con microscopía electrónica. Para realizar los ensayos de comportamiento mecánico, según la norma ASTM A-370, se realizó corte con agua para obtener la geometría requerida en las probetas. Se utilizó una máquina universal de ensayos Shimadzu_UH_X con control de desplazamiento y se ajustó una velocidad de ensayo de $5,0 \mathrm{~mm} /$ min. Las superficies de fractura resultantes fueron observadas utilizando de nuevo microscopía MEB. La dureza de las fases fue evaluada utilizando un microdurometro marca Leco 402 utilizando una carga de $500 \mathrm{~g}$ y tiempo de $10,0 \mathrm{~s}$.

\section{RESULTADOS Y DISCUSIÓN}

\section{Influencia del revenido en la microestructura}

La Figura 1 muestra las imágenes MEB del acero DP580 en el estado inicial. Se puede apreciar la microestructura de fase dual compuesta por islas interconectadas de martensita rodeadas de una matriz de ferrita. La medición y análisis estadístico sobre diez imagenes de la fracción de las fases presentes dio como resultado promedio un $12 \%$ de fracción volumétrica de martensita, este resultado es consecuente con el contenido de carbono de $0,069 \%$.

La Figura 2 muestra las imágenes MEB del acero después del tratamiento de revenido a 150 , a 300 y a $500{ }^{\circ} \mathrm{C}$. En el acero revenido a $150{ }^{\circ} \mathrm{C}$, se observa que la distribución de la martensita en islas interconectadas difiere muy poco de la microestructura del acero sin tratamiento. En las muestras revenidas a 300 y a $500{ }^{\circ} \mathrm{C}$ se observa el aparecimiento progresivo de discontinuidades longitudinales en la fase martensita. Este fenómeno obedece a que el tratamiento térmico de revenido genera la segregación por difusión del carbono en las dislocaciones con la consecuente formación de precipitados de carburos de hierro $\varepsilon$ y de cementita. Este fenómeno observado en la fase martensita fue reportado también por Gündüz [20] en un estudio sobre la trasformación de la martensita y su influencia en las propiedades mecánicas.

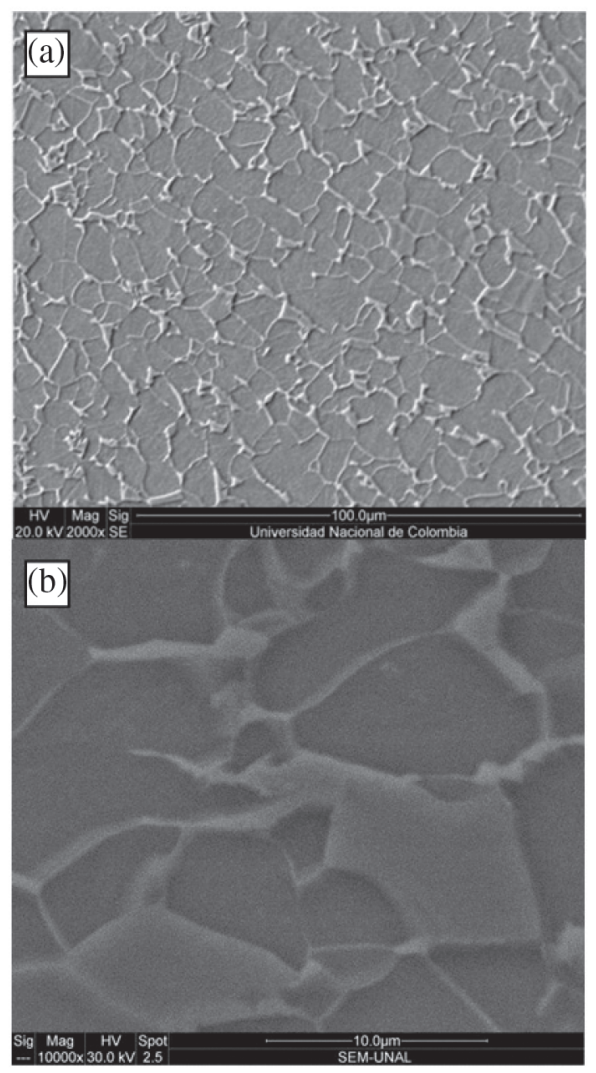

Figura 1. (a) Imágen MEB del acero DP580 en estado inicial, (b) ampliación en detalle de la fase martensita.

Las reacciones de revenido de los aceros de fase dual son una combinación de los efectos sinérgicos del revenido de cada una de las fases individuales. Debido a las moderadas temperaturas de revenido que se utilizaron, la estructura básica ferritamartensita se mantuvo. La fracción volumétrica de martensita presentó una leve disminución con el incremento de la temperatura de revenido (hasta $9 \%$ en las probetas tratadas a $500{ }^{\circ} \mathrm{C}$ ). Lo anterior se debe a que el tratamiento térmico de revenido produce transformaciones de la fase martensita [1], especialmente difusión del carbono de esta fase, tal como fue señalado por Fang y colaboradores [19].

\section{Influencia del revenido en el comportamiento mecánico.}

La Figura 3 ilustra las curvas esfuerzo-deformación de ingeniería obtenidas en los ensayos de tracción uniaxial según la norma ASTM A-370. La probeta sin tratamiento térmico y aquella recocida a $150{ }^{\circ} \mathrm{C}$ presentan una fluencia continua, debido 


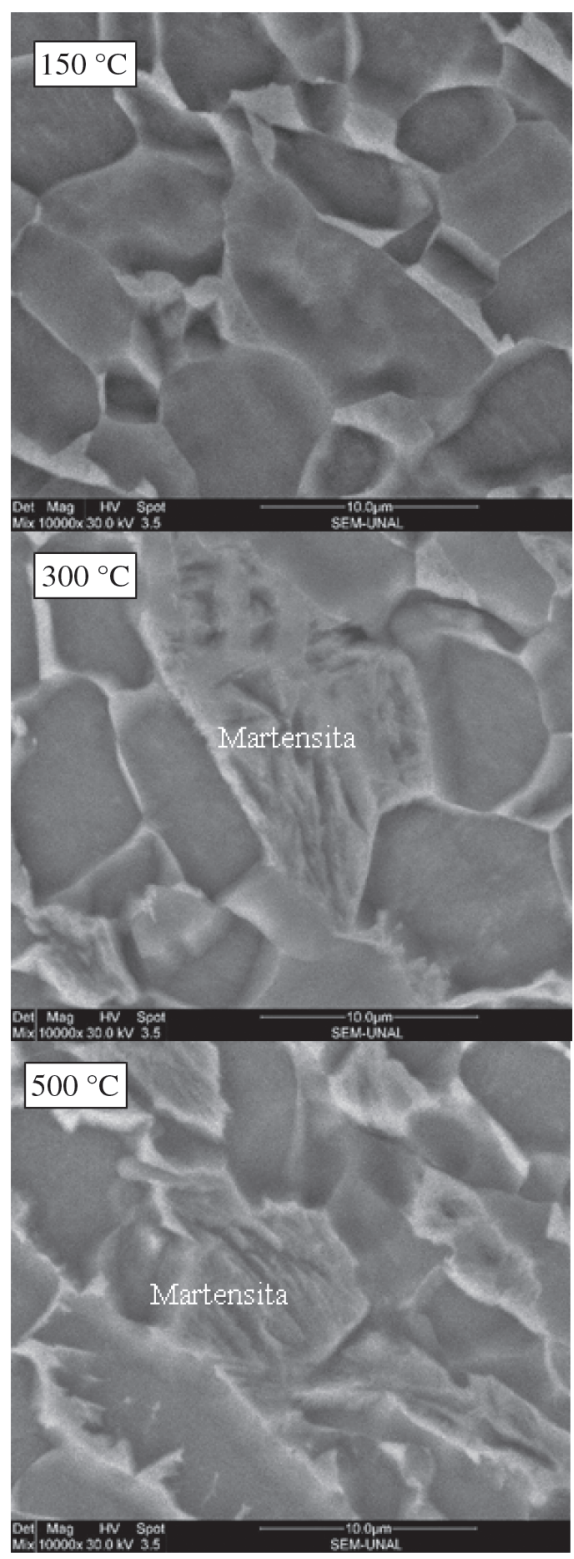

Figura 2. Micrografías MEB del acero DP580 después de revenido a 150,300 y $500{ }^{\circ} \mathrm{C}$.

esencialmente a la presencia de ferrita con alta cantidad de dislocaciones no bloqueadas producidas durante la trasformación de la austenita en martensita en el proceso de obtención inicial del acero dual [21-22].

La fluencia discontinua con picos múltiples observada en las probetas revenidas a temperaturas de $200^{\circ} \mathrm{C}$ y mayores se pueden explicar debido al aumento del bloqueo al movimiento de las dislocaciones en

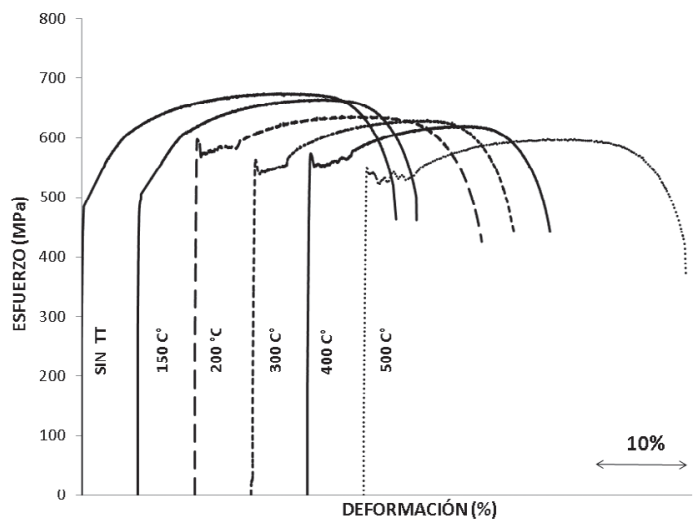

Figura 3. Curvas esfuerzo-deformación de ingeniería de las probetas sin tratamiento y de las revenidas.

la fase ferrita producido por el carbono difundido desde la fase martensita por efecto del proceso de revenido $[16,18]$.

En la figura 4 se presentan los resultados del esfuerzo último y del esfuerzo de fluencia en función de la temperatura de revenido. En las probetas con fluencia discontinua, la resistencia a la fluencia fue tomada como el promedio de los picos máximos y mínimos que se presentaron en la zona de fluencia. El cálculo de la fluencia en las probetas que presentaron fluencia continua se estimó directamente al $0.2 \%$ de deformación.

En las probetas revenidas hasta $200^{\circ} \mathrm{C}$, la resistencia a fluencia aumenta para disminuir suavemente a

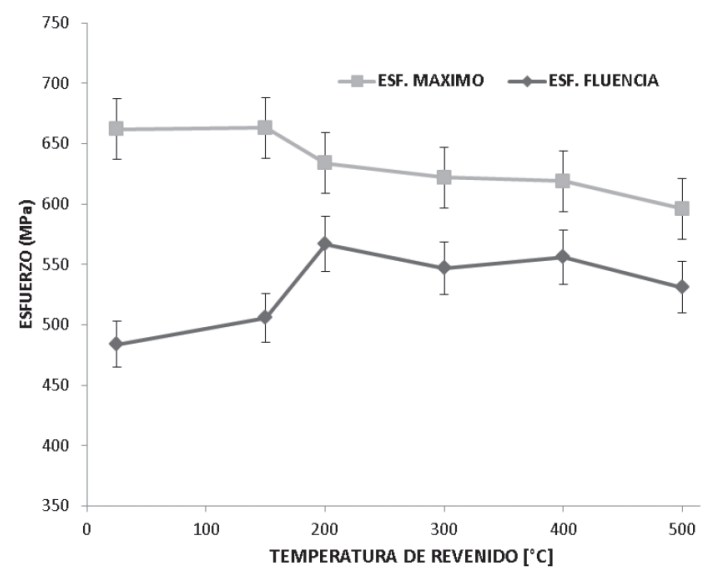

Figura 4. Esfuerzo último y esfuerzo de fluencia en función de la temperatura para una hora de revenido del acero DP580. 
partir de esta temperatura. En las probetas revenidas hasta $150{ }^{\circ} \mathrm{C}$ se observa que la resistencia última se mantiene para disminuir a partir de esta temperatura. El incremento inicial en la resistencia a fluencia se puede atribuir a la formación de carburos finos de hierro y a que el carbono difundido de la fase martensita a la fase ferrita bloquea las dislocaciones libres en la ferrita. A temperaturas de revenido por encima de $200{ }^{\circ} \mathrm{C}$ los esfuerzos residuales disminuyen debido a la disminución en volumen de la fase martensita, dando como resultado una disminución en la resistencia a fluencia del acero dual [18, 21, 24].

Al incrementar la temperatura de revenido la concentración de carbono en la fase martensita continúa disminuyendo debido a la difusión de sus átomos de carbono. Según la hipótesis presentada por Lou y Northwood [25], este fenómeno se da por el aumento de la difusividad del carbono con la temperatura y/o por la alta concentración de átomos de soluto en la red de dislocaciones en el hierro $\alpha$ a altas temperaturas.

En los aceros duales la resistencia mecánica depende principalmente de las propiedades de la fase martensita $[11,15]$. La disminución en la resistencia última al incrementar la temperatura de revenido, observada en la Figura 4, está directamente relacionada con la transformación de la martensita por efecto del proceso de revenido [1]. Hasta $\operatorname{los} 200^{\circ} \mathrm{C}$ se presenta precipitación de carburos de hierro $\alpha$ y pérdida parcial de tetragonalidad de la martensita. Entre 200 y $300{ }^{\circ} \mathrm{C}$ se presenta descomposición de la austenita retenida. Entre 200 y $350{ }^{\circ} \mathrm{C}$ se presenta sustitución de los carburos de hierro $\alpha$ por cementita y la martensita pierde completamente la tetragonalidad. Por encima de $350{ }^{\circ} \mathrm{C}$ la cementita presente en la martensita revenida se engruesa y se esferoidiza [1]. El resultado de estas transformaciones sucesivas explica la disminución en la resistencia última y un posible aumento de la ductilidad de la martensita revenida.

El efecto del proceso de revenido sobre la microdureza de las fases martensita y ferrita del acero estudiado se ilustra en la Figura 5. Puede observarse que la dureza de la fase martensita disminuye levemente para temperaturas de tratamiento superiores a $200{ }^{\circ} \mathrm{C}$ y que no hay cambios en la dureza de la fase ferrita. Luo y colaboradores [15] atribuyen la disminución en la dureza de la fase martensita a la difusión de

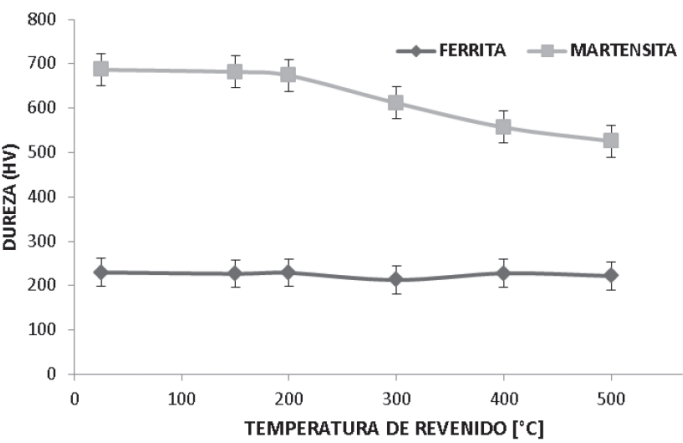

Figura 5. Efecto del revenido sobre la microdureza de la martensita y de la ferrita en el acero DP580.

carbono hacia la fase ferrita y a la disminución de los esfuerzos residuales por la contracción de esta fase. Posiblemente también influye la perdida de continuidad de la fase martensita observada en la Figura 2 para temperaturas mayores a $300{ }^{\circ} \mathrm{C}$.

\section{Análisis de fractura}

La Figura 6 muestra la superficie de fractura del acero DP580 sin revenido, revenido a $150{ }^{\circ} \mathrm{C}$, revenido a $300^{\circ} \mathrm{C}$ y revenido a $500^{\circ} \mathrm{C}$. En el acero sin tratamiento térmico (Figura 6a y 6b) se observa la superficie típicamente rugosa de una fractura dúctil.

En la superficie de fractura del acero revenido a $150{ }^{\circ} \mathrm{C}$ (Figura 6c y 6d) se evidencian más zonas de clivaje como resultado de la interacción entre las dislocaciones y átomos carbono y/o precipitados; esto evidencia una leve disminución en la ductilidad del acero. La superficie fractura de probetas tratadas a $300{ }^{\circ} \mathrm{C}$ (Figura 6e y $6 f$ ) exhibe una recuperación de la ductilidad evidenciado por el tamaño y mayor cantidad de micro-agujeros. En las probetas revenidas a $500{ }^{\circ} \mathrm{C}$ (Figura $6 \mathrm{~g}$ y $6 \mathrm{~h})$ no se aprecian diferencias sustanciales con las muestras revenidas a $300^{\circ} \mathrm{C}$. Tosun y Gündüz [23] y Chang [24] concluyen que en este tipo de aceros la ductilidad depende principalmente del porcentaje y características estructurales de fase ferrita, aunque como mencionado atrás el aumento de temperatura del proceso de revenido también propicia un aumento de la ductilidad de la martensita revenida.

\section{CONCLUSIONES}

Con el desarrollo del presente trabajo se pude concluir que los efectos del tratamiento térmico 


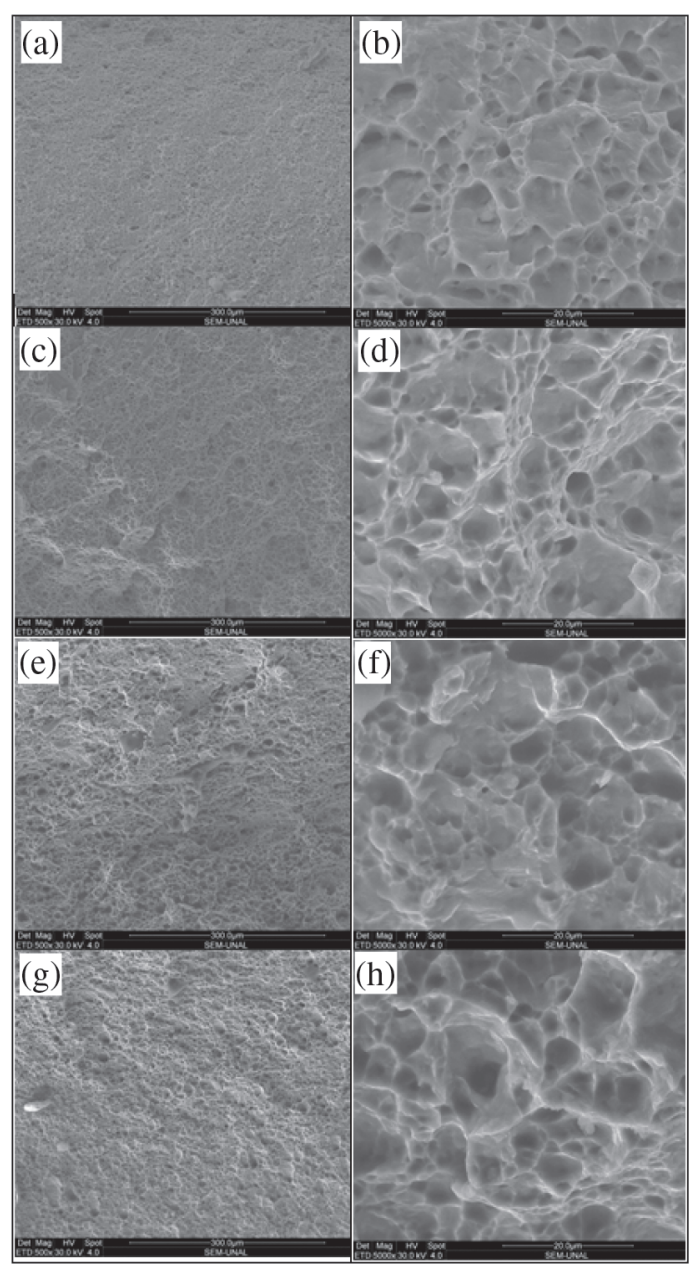

Figura 6. Superficie de fractura del acero DP580, (a) y (b) sin revenido, (c) y (d) revenido a $150{ }^{\circ} \mathrm{C}$, (e) y (f) revenido a $300^{\circ} \mathrm{C},(\mathrm{g})$ y (h) revenido a $500{ }^{\circ} \mathrm{C}$.

de revenido sobre el acero de fase dual DP580 analizado son consecuencia de un efecto sinérgico entre las transformaciones en la fase martensita y en la fase ferrita. Temperaturas de revenido variando entre 150 y $500{ }^{\circ} \mathrm{C}$ afectan de manera gradual la microestructura, permitiendo la obtención de diferentes estados de resistencia y ductilidad. Este proceso permite entonces adaptar el material a los requerimientos de la aplicación.

En los ensayos de tracción uniaxial se evidenciaron diferencias en función de la temperatura de revenido. En las probetas sin tratamiento térmico y revenidas a $150{ }^{\circ} \mathrm{C}$ se presentó fluencia continua debido a la presencia de una fase ferrita con elevada cantidad de dislocaciones no bloqueadas. El proceso de revenido produjo un aumento del bloqueo producido por el carbono difundido de la fase martensita a la fase ferrita. Esto se evidencia en la fluencia discontinua para las probetas revenidas a temperaturas superiores a $200{ }^{\circ} \mathrm{C}$.

A temperaturas de revenido iguales o superiores a $300{ }^{\circ} \mathrm{C}$ las islas de fase martensita presentaron mayor discontinuidad comparadas con la distribución de la fase martensita del acero sin tratamiento, este fenómeno se debe a que el tratamiento térmico de revenido genera difusión del carbono, provocando la segregación del mismo en las dislocaciones y la precipitación de carburos finos de hierro $\varepsilon$ y de cementita. Lo anterior influye en la disminución de la resistencia última, pues en el acero dual estudiado, la resistencia mecánica depende principalmente de las propiedades de la fase martensita.

El ablandamiento de la martensita con el revenido reduce la resistencia, de otro lado el leve incremento de la ductilidad en la martensita no da lugar a un aumento significativo en la ductilidad del acero de fase dual. Cabe señalar que la principal fuente de buena ductilidad en aceros de fase dual es la presencia de ferrita blanda, es decir que el porcentaje en volumen de ferrita controla la ductilidad de los aceros de fase dual.

\section{AGRADECIMIENTOS}

Los autores agradecen a la Vicerrectoría de Investigación de la Universidad Nacional de Colombia por el apoyo financiero para el desarrollo del presente proyecto.

\section{REFERENCIAS}

[1] R.W.K. Honeycombe and H.K.D.H. Bhadeshia. "Steels, microstructure and properties". $3^{\text {th }}$ edition. Butterworth-Heinemann. London, England. 2006. ISBN: 13:978-0-750-68084-4.

[2] S. Robertson. "Unlocking the world of dualphase, trip and twip steels". American metal market, pp. 54-55. 2007. Fecha de consulta: 17 de Julio de 2013. URL: http://www.integpg. com/hsmm/files/AMM_TechTrak_Aug07. pdf. ISSN: 0002-9998.

[3] A. Grajcar, R. Kuziak and W. Zalecki. "Third generation of AHSS with increased fraction 
of retained austenite for the automotive industry". Archives of civil and mechanical engineering. Vol. 12, Issue 3, pp. 334-341. 2012. ISSN: 1644-9665. DOI: $10.1016 /$ j. acme.2012.06.011.

[4] R.G. Davies. "Fundamentals of dual phase steels". Conference proceedings (Metallurgical society of AIME), pp. 265277. Warrendale, PA, USA. 1981. ISBN: 10:0895203839.

[5] G.R. Speich. "Fundamentals of dual phase steels". Conference proceedings (Metallurgical society of AIME), pp. 3-45. Warrendale, PA, USA. 1981. ISBN: 10:0895203839.

[6] S. Sun and M. Pugh. "Properties of thermomechanically processed dual-phase steels containing fibrous martensite". Materials science and engineering A. Vol. 335, Issue 1-3, pp. 298-308. 2002. ISSN: 09215093. DOI: 10.1016/S0921-5093(01)01942-6.

[7] G. Jhaa, S. Dasa, A. Lodha and A. Haldarb ."Development of hot rolled steel sheet with $600 \mathrm{MPa}$ UTS for automotive wheel application". Materials science and engineering: A. Vol. 552, pp. 457-463. 2012. ISSN: 0921-5093. DOI: $10.1016 / \mathrm{j}$. msea.2012.05.070.

[8] M. Erdogan and R. Priestner. "Effect of martensite content, its dispersion, and epitaxial ferrite content on Bauschinger behaviour of dual phase steel". Journal of materials science and technology. Vol. 18, pp. 369-376. 2002. ISSN: 1005-0302. DOI: $10.1179 / 026708302225001679$.

[9] M. Sarwar, E. Ahmad, K.A. Qureshi and T. Manzoor. "Influence of epitaxial ferrite on tensile properties of dual phase steel". Materials design. Vol. 28, pp. 335-40. 2007. ISSN: 0261-3069. DOI: $10.1016 / \mathrm{j}$. matdes.2005.05.019.

[10] S.H.M. Anijdan and H. Vahdani. "Roomtemperature mechanical properties of dual-phase steels deformed at high temperatures". Materials letters. Vol. 59, pp. 1828-1830. 2005. ISSN: 0167-577X. DOI: 10.1016/j.matlet.2004.08.046.

[11] V. Tarigopula, O.S. Hopperstad, M. Langseth and A.H. Clausen. "Elastic-plastic behaviour of dual-phase, high-strength steel under strain-path changes". European journal of mechanics A - Solids. Vol. 27 Issue 5, pp. 764-782. 2008. ISSN: 09977538. DOI: 10.1016/j.euromechsol.2008.01.002.

[12] H. Palkowski and T. Anke. "Bake hardening of hot rolled multiphase steels under biaxial pre-strained conditions". Steel research international. Vol. 77, pp. 675-9. 2006. ISSN: 1611-3683.

[13] G.T. Eldis. "The Influence of microstructure and testing procedure on the measured mechanical properties of heat treated dualphase steels". Conference proceedings (Metallurgical Society of AIME), pp. 310316. Warrendale, PA, USA. 1981. ISBN: 10:0895203839

[14] M.S. Rashid and B.V.N. Rao. "Tempering characteristics of a vanadium containing dualphase steel". Metallurgical transactions A. Vol. 13, Issue 10, pp 1679-1686. 1982. ISSN: 1073-5623. DOI: 10.1007/BF02647823.

[15] J.J. Luo, W. Shi, Q.F. Huang and L. Li. "Heat treatment of cold-rolled low-carbon Si-Mn dual phase steel". Journal of iron and steel research international. Vol. 17, Issue 1, pp. 54-58. 2010. ISSN: 1006-706X. DOI: 10.1016/S1006-706X(10)60045-3.

[16] H. Matsuda, R. Mizuno, Y. Funakawa, K. Seto, S. Matsuoka and Y. Tanaka. "Effects of auto-tempering behaviour of martensite on mechanical properties of ultra high strength steel sheets". Journal of alloys and compounds. Vol. 577, Issue 15, pp. S661-S667. 2013. DOI: 10.1016/j.jallcom.2012.04.108.

[17] S. Gündüz. "Static strain ageing behaviour of dual phase steels". Materials science and engineering A. Vol. 486, pp. 63-71. 2008. ISSN: 0921-5093. DOI: 10.1016/j. msea.2007.08.056.

[18] A.J. Abdalla, L.R.O. Hein, M.S. Pereira and T.M. Hashimoto. "Mechanical behaviour of strain aged dual phase steels". Materials science technology. Vol. 15, pp. 1167-70. 1999. ISSN: 0267-0836. DOI: 10.1179/026708399101505211.

[19] X. Fang, Z. Fan, B. Ralph, P. Evans and R. Underhill. "Effects of tempering temperature on tensile and hole expansion properties of a C-Mn steel". Journal of materials processing \& technology. Vol. 132, Issue 1-3, pp. 215218. 2003. ISSN: 0924-0136. DOI: $10.1016 /$ S0924-0136(02)00923-8. 
[20] S. Gündüz. "Effect of chemical composition, martensite volume fraction and tempering on tensile behaviour of dual phase steels". Materials letters. Vol. 63, pp. 2381-2383. 2009. ISSN: 0167-577X. DOI: $10.1016 / j$. matlet.2009.08.015.

[21] F. Ozturk, S. Toros and S. Kilic. "Tensile and spring-back behavior of DP600 advanced high strength steel at warm temperatures". Journal of iron and steel research international. Vol. 16, Issue 6, pp. 41-46. 2009. ISSN: 1006-706X. DOI: 10.1016/S1006-706X(10)60025-8.

[22] R.O. Rocha, T.M.F. Melo, E.V. Pereloma and D.B. Santos. "Microstructural evolution at the initial stages of continuous annealing of cold rolled dual-phase steel". Materials science and engineering A. Vol. 391, pp. 296-304.
2005. ISSN: 0921-5093. DOI: 10.1016/j. msea.2004.08.081.

[23] A. Tosun and S. Gündüz. "Influence of straining and ageing on the room temperature mechanical properties of dual phase steel". Materials design. Vol. 29, pp. 1914-1918. 2008. ISSN: 0261-3069. DOI: 10.1016/j. matdes.2008.04.028.

[24] P. Chang. "Temper-aging of continuously annealed low carbon dual phase steel". Metallurgical transactions A. Vol. 15, pp. 73-86. 1984. ISSN: 1073-5623. DOI: 10.1007/BF02644389.

[25] S. Lou and D.O. Northwood. "Effect of temperature on the lower yield strength and static strain ageing in low-carbon steels". Journal of materials science. Vol. 30, pp. 1434-1438. 1995. ISSN: 0022-2461. DOI: 10.1007/BF00375244. 